\title{
Interparticle Electromagnetic Coupling in Assembled Gold-Necklace Nanoparticles
}

\author{
Guda Ramakrishna $^{\dagger}$, Qiu Dai ${ }^{\dagger \dagger}$, Jianhua Zou ${ }^{\dagger \dagger}$, Qun Huo ${ }^{\dagger \dagger}$ and Theodore Goodson ${ }^{\star \dagger}$ \\ Department of Chemistry, University of Michigan, Ann Arbor, MI, 48109, and \\ ${ }^{+\dagger}$ Nanoscience Technology Center and Department of Chemistry, University of Central \\ Florida, Orlando FL 32826
}

\section{Supporting information}

\section{Complete reference of $\mathbf{1 6 b}$}

Arbouet, A.; Voisin, C.; Christofilos, D.; Langot, P; Del Fatto, N.; Valle, F.; Lerme, J.;

Celep, G.; Cottanacin, E.; Gaudry, M.; Pellarin, M.; Broyer, M.; Maillard, M.; Pileni, M. P.; Treguer, M. Phys. Rev. Lett. 2003, 90, 177401.

Synthesis of monocarboxylated gold nanoparticles and nano-necklace particles: The synthesis and characterization of the gold nanoparticle necklaces was initially reported in ref 1 and the monocarboxylatedgold nanoparticles to synthesize them are published in ref 2. Control experiment has been performed to confirm that the necklace structure was due to covalent attachment of nanoparticles to the polylysine and ring closure of polymer chain. As control experiment, we coupled monofunctional nanoparticles with protected polylysine and polyacrylic acid (which cannot cyclize into ring structure). In both cases, we did not see any necklace structures. Shown in Figure S1 are the TEM measurements that have been carried out to characterize the necklace particles that are used for ultrafast measurements. We observed major fraction of the necklace particles and the necklace sizes are around 20 to $70 \mathrm{~nm}$. Complete details of the characterization are given in ref 1 . 

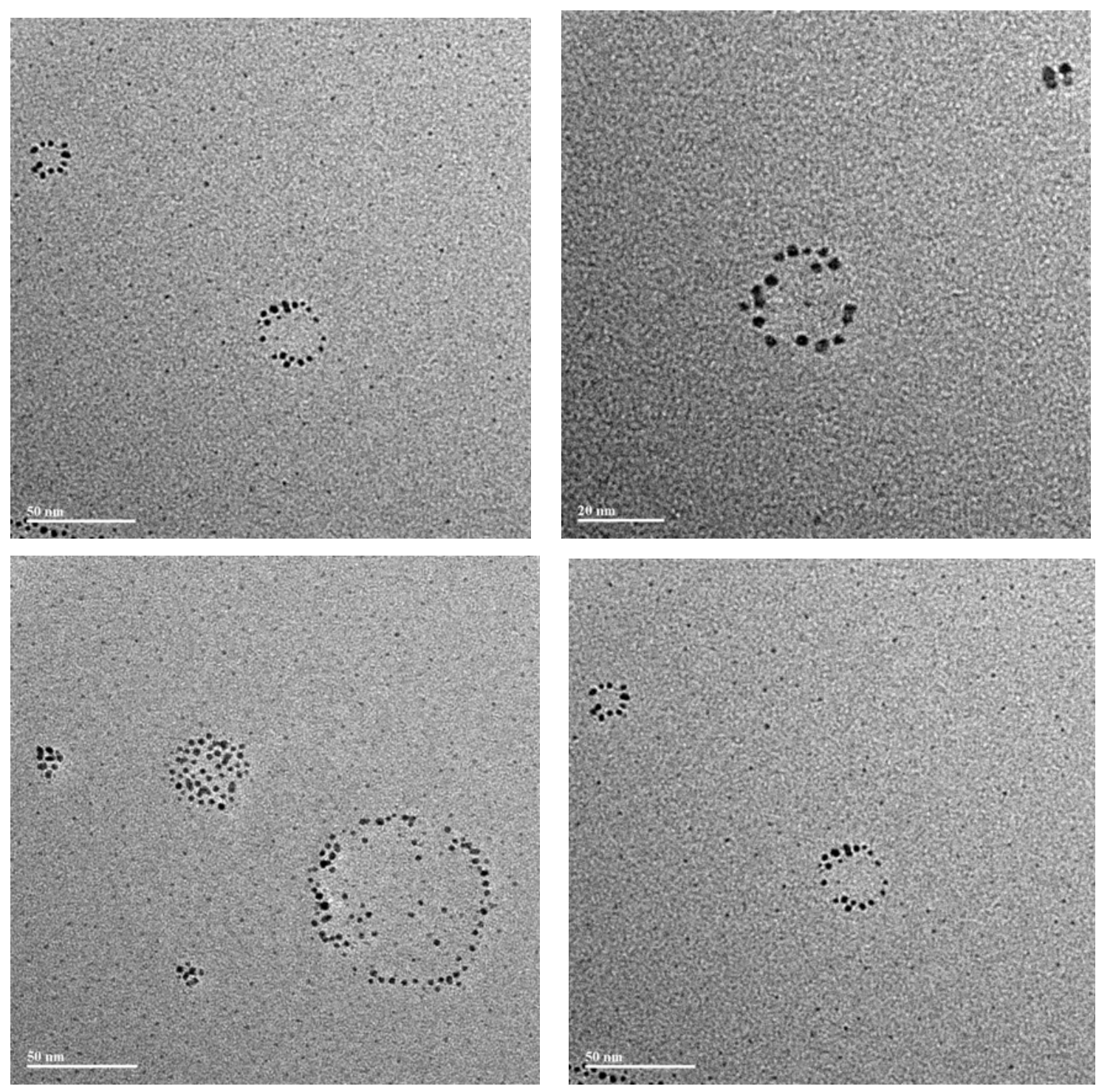

Figure S1: Some of the transmission electron microscopy images of gold-necklace (goldpolylysine hybrid) particles.

\section{Transient absorption measurements}

Femtosecond transient absorption investigations have been carried out using ultrafast pump-probe spectrometer detecting in the visible region. Briefly, $1 \mathrm{~mJ}, 100 \mathrm{fs}$ pulses at $800 \mathrm{~nm}$ with a repetition rate of $1 \mathrm{KHz}$ were obtained from Nd:YLF(Empower) pumped Ti:Sapphire regenerative amplifier (Spitfire ${ }^{\circledR}$, Spectra-Physics) with the input from Millennia pumped Ti:Sapphire oscillator (Spectraphysics, Tsunami). The output of laser beam was split to generate pump and probe beam pulses with a beam splitter ( $85 \%$ and $15 \%$ ). The pump beam was produced by an optical parametric amplifier (OPA- 
800C). The pump beams used in the present investigation, i.e., 375, $430 \mathrm{~nm}$, were obtained from the fourth harmonic of the signal and idler beams respectively. They were focused onto the sample cuvette. The probe beam was delayed with a computer controlled motion controller and then focused into a $2 \mathrm{~mm}$ sapphire plate to generate white light continuum. The white light was then overlapped with the pump beam in a 2 mm quartz cuvette containing the sample and the change in absorbance for the signal was collected by a CCD detector (Ocean optics). Data acquisition was controlled by the software from Ultrafast systems. Typical power of probe beam was $\sim 10 \mu \mathrm{J} / \mathrm{cm}^{2}$ while the pump beam was around $1000 \mu \mathrm{J} / \mathrm{cm}^{2}$. Magic angle polarization was maintained between the pump and probe using a wave plate. Pulse duration was obtained from the nonresonant fitting of the solvent response, which was around 120 fs. The sample was stirred by a rotating magnetic stirrer and little degradation of the sample was observed during the experiments.

Parts A and B of Figure S2 show the transient absorption spectra of Au-neck from 100 fs to 800 fs and 800 fs to 10 ps respectively. It can be observed from Figure 2A that both the positive absorption as well as bleach of surface plasmon absorption grows with increase in time delay. This gives us the information about the slower electron-electron scattering rate of Au-neck particles. However, after electron-electron scattering is complete both excited state absorption and bleach decay with time indicating the electron-phonon relaxation dynamics.
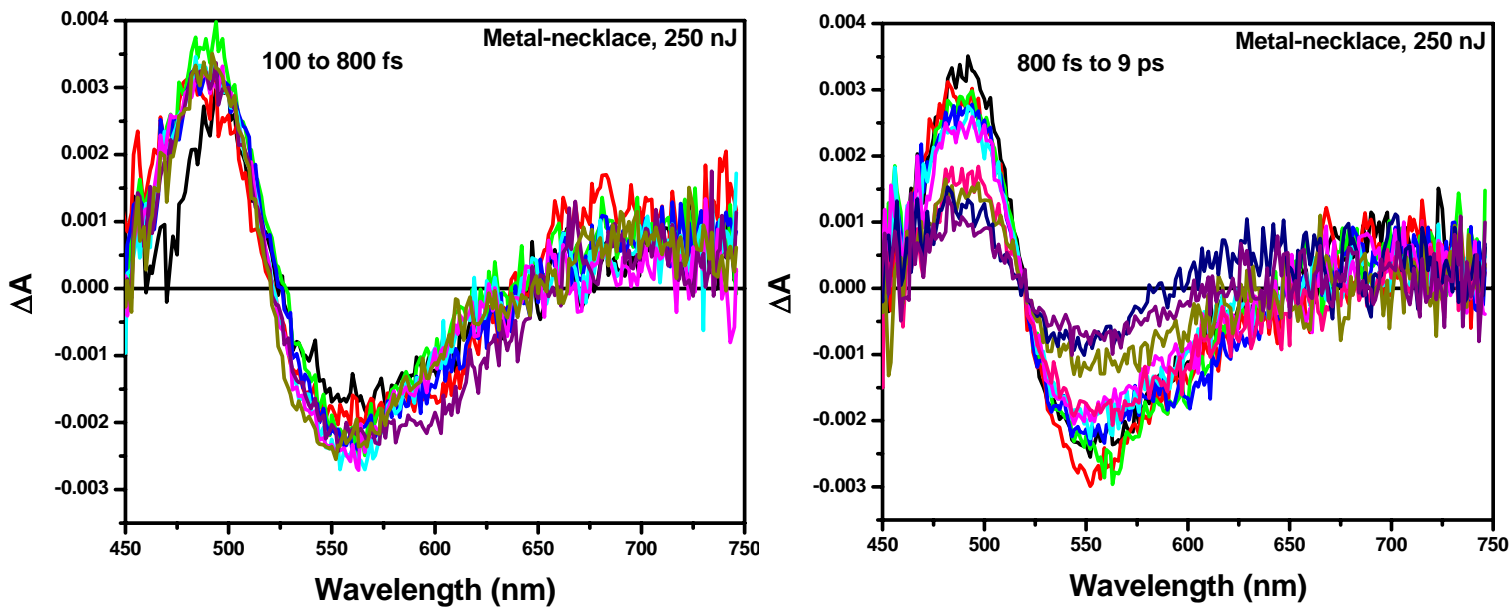

Figure S2: Transient absorption spectra at different time delays (a) $100 \mathrm{fs}$ to $800 \mathrm{fs}$ (b) 800 fs to 10 ps of Au-Neck particles dissolved in Methanol + Dichoromethane + 1\%TFA mixture. 
In contrast no such growth of surface plasmon bleach is observed for Au-Np suggesting a faster electron-electron scattering (Figure S3).

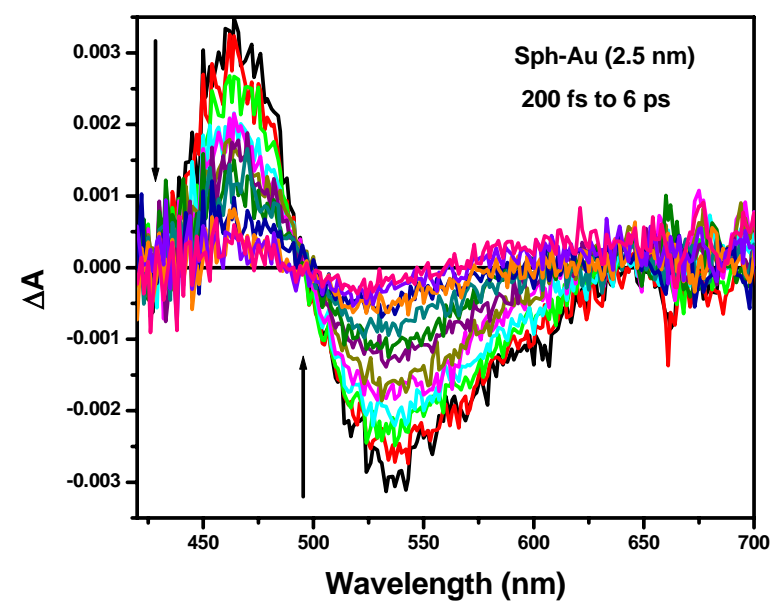

Figure S3: Transient absorption spectra at different time delay of spherical gold nanoparticles after excitation at $390 \mathrm{~nm}$ with $230 \mathrm{~nJ}$ laser power.

\section{Pump-power dependent measurements:}

Transient absorption measurements with varying pump power have been carried out to understand the effect of pump power on electron-electron scattering and electronphonon relaxation. We have determined the electron-electron thermalization $\left(\tau_{e-e}\right)$ time by fitting the measured signal as a function of mono-exponential rise and using a phenomenological response function of the form used by Vallee and co-workers ${ }^{3,4}$.

$F(t)=u(t)\left[1-\exp \left(-t / \tau_{e-e}\right)\right] \exp \left(-t / \tau_{e-p h}\right)$

Where $\tau_{e-p h}$ is the electron-phonon relaxation time and $u(t)$ is the step function. The decay signal $F(t)$ is convoluted with the instrument response function to obtain the electronelectron scattering and electron-phonon thermalization times. Thus, we have re obtained the electron-electron thermalization times of spherical gold nanoparticles and Goldnecklace particles. Obtained values stand as 560 to $680 \mathrm{fs}$ and vary little bit as a function of pump-power. Analysis of the data of gold-necklace particles still had given higher 
electron-electron thermalization times than what has been observed for spherical gold nanoparticles (in the order of $200 \pm 80 f s$ only).
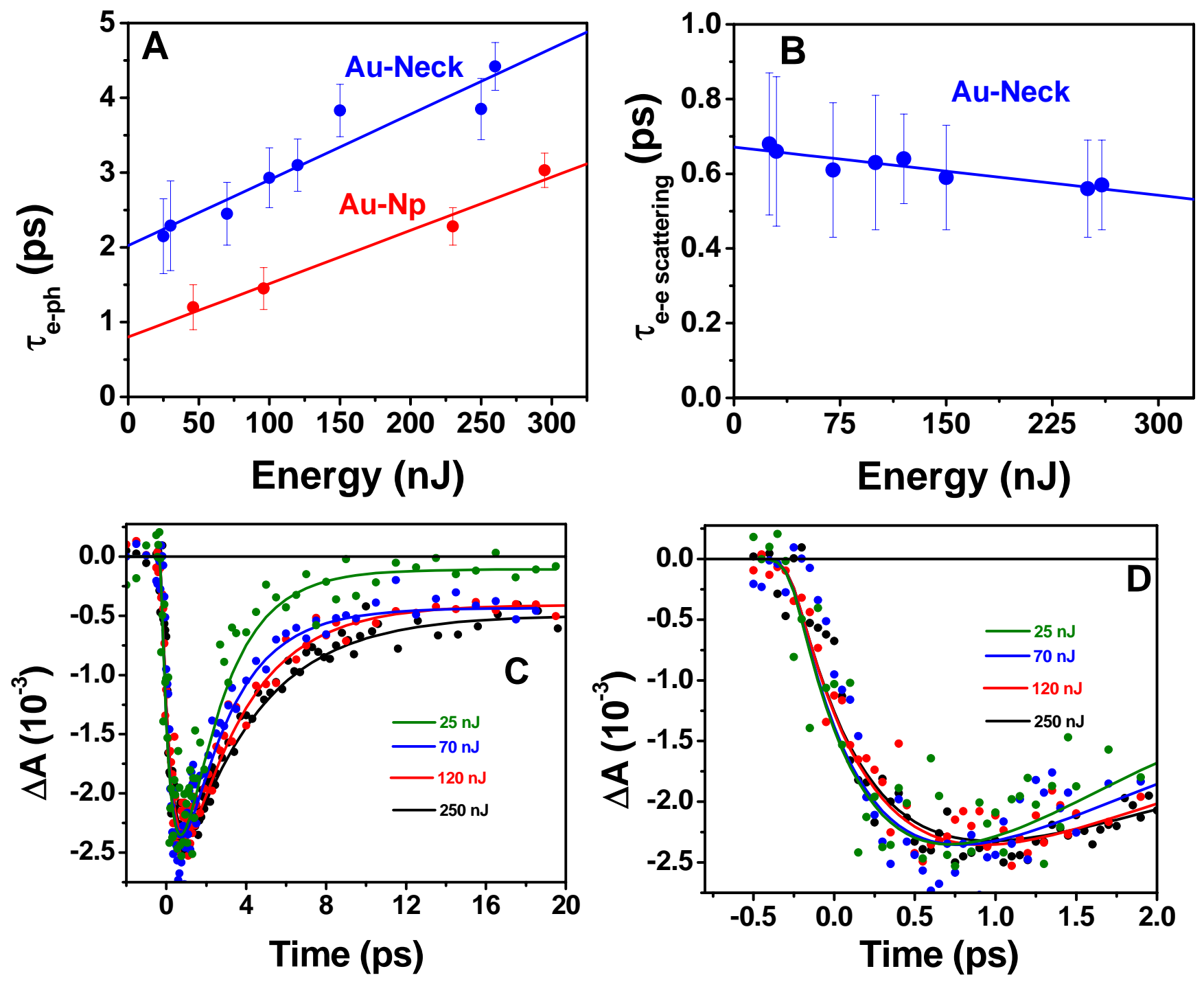

Figure S4. A. Power dependent electron-phonon relaxation times of spherical $\mathrm{Au}-\mathrm{Np}$ and $\mathrm{Au}-$ Neck. B. Electron-electron scattering time plot of Au-Neck particles. C. Plot of bleach kinetics at $550 \mathrm{~nm}$ for Au-Neck at pump powers (25 nJ to $250 \mathrm{~nJ}$ ) showing electron-phonon relaxation and D. Bleach kinetic traces showing electron-electron scattering.

After careful analysis, we observed that the e/e scattering is very weakly dependent (650 fs to $540 \mathrm{fs}$ with increase in pump power from $25 \mathrm{~nJ}$ to $260 \mathrm{~nJ}$ ) on pump power while e/p relaxation is very much dependent on pump-power (2.25 ps to 4.3 ps with increase in pump power from $25 \mathrm{~nJ}$ to $260 \mathrm{~nJ}$ ) and this might be due to the fact that they happen at two different time domain and the inter particle electromagnetic coupling modifies their pump power dependence. In isolated gold nanoparticles, the e/e scattering 
is an intra particle phenomenon wherein the hot electrons share their energy among the manifold of electrons within the particle whose time scale is in the order of 100 to $300 \mathrm{fs}$ and decreases with pump power (Ref 16 of manuscript). However, in the case of present system, since the gold particles are arranged closely and in a circular fashion, along with the intra-particle phenomenon one should consider the inter-particle e/e relaxation which takes makes it to be longer. As the e/e relaxation also involves an inter particle phenomenon, the population of electrons within the particle and the neighboring particles are involved and because of this the temperature raise induced by the increase in pumppower and their consequent energy sharing with the other electrons found to have a weak impact and hence there is a very weak pump power dependence. However, the e/p relaxation is dependent on pump power since the population of hot electrons in the system varies with the pump power and its relaxation to the lattice phonons does depend on pump power. Power zero electron phonon relaxation dynamics has been obtained extrapolating the decay time constants obtained at different pump powers from global fit analysis. Time constant of $2.01 \pm 0.3$ ps is quite high than what has been obtained for spherical gold nanoparticles of different sizes ( $\sim 0.7 \mathrm{ps}$ ). The longer electron-phonon relaxation time can be ascribed to the effective electronic coupling between several gold nanoparticles as they are oriented close to each other.

\section{References}

1. (a) Worden, J. G.; Shaffer, A. W.; Huo, Q. Chem. Commun. 2004, 518. (b) Dai, Q.; Worden, J. G.; Trullinger, J.; Huo, Q. J. Am. Chem. Soc. 2005, 127, 8008.

2. Liu, X.; Worden, J. G.; Dai, A.; Zou, J.; Wang, J.; Huo, Q. Small 2006, 2, 1126.

3. (a) Voisin, C.; Christofilos, D.; Del Fatti, N.; Vallee, F.; Prevel, B.; Cottancin, E.; Lerme, J.; Pellanin, M.; Broyer, M. Phys. Rev. Lett. 2000, 85, 2200.

4. (a) Voisin, C.; Christofilos, D.; Loukakos, P. A.; Del Fatti, N.; Vallee, F.; Lerme, J.; Faudry, M.; Cottanacin, E.; Pellarin, M.; Broyer, M.; Phys. Rev. B. 2004, 69, 165416. (b) Arbouet, A.; Voisin, C.; Christofilos, D.; Langot, P; Del Fatto, N.; Valle, F.; Lerme, J.; Celep, G.; Cottanacin, E.; Gaudry, M.; Pellarin, M.; Broyer, M.; Maillard, M.; Pileni, M. P.; Treguer, M. Phys. Rev. Lett. 2003, 90, 177401. 